\title{
On the Sperner property and Gorenstein Algebras Associated to Matroids
}

\author{
Toshiaki Maeno $\|^{1}$ and Yasuhide Numata ${ }^{23}$ \\ ${ }^{1}$ Department of Electrical Engineering, Kyoto University, Japan \\ ${ }^{2}$ Department of Mathematical Informatics, The University of Tokyo, Japan \\ ${ }^{3}$ Japan Science and Technology Agency (JST) CREST
}

\begin{abstract}
We introduce a certain class of algebras associated to matroids. We prove the Lefschetz property of the algebras for some special cases. Our result implies the Sperner property for the Boolean lattice and the vector space lattice.

Résumé. Nous présentons une classe d'algèbres associées aux matroïdes. Nous démontrons que dans quelques cas spécifiques, ces algèbres verifient la propriété de Lefschetz. Notre résultat implique la propriété de Sperner pour l'algèbre de Boole et pour le poset d'espace vectoriel.
\end{abstract}

Keywords: Lefschetz property, ranked poset, universal Gröbner bases.

\section{Introduction}

The strong Lefschetz property for Artinian Gorenstein algebras is a ring-theoretic interpretation of the Hard Lefschetz Theorem for compact Kähler manifolds. Stanley developed the ideas of applications of the Lefschetz property to combinatorial problems. For example, he proved the Sperner property of the Bruhat ordering on the Weyl groups by the Hard Lefschetz Theorem for the flag varieties in his paper [15]. One of the main topics of this article is an application of the Lefschetz property for a certain kind of Gorenstein algebras to the Sperner property of the ranked posets associated to some matroids. Our result contains the Boolean lattice $2^{[n]}$, i.e., the set of the subsets of $\{1, \ldots, n\}$ ordered by the inclusion, and the vector space lattice $V(q, n)$, the set of the linear subspaces of the vector space $\mathbb{F}_{q}^{n}$ ordered by the inclusion.

In [14], Sperner determined maximum-sized antichains of the Boolean lattice $2^{[n]}$. His work is the origin of the theory of the Sperner property. It is known that the Sperner property of the Boolean lattice is proved also from the strong Lefschetz property of the algebra $k\left[x_{1}, \ldots, x_{n}\right] /\left(x_{1}^{2}, \ldots, x_{n}^{2}\right)$. For example, we can obtain the strong Lefschetz property of the algebra by applying the Hard Lefschetz Theorem to the product $\left(\mathbb{P}^{1}\right)^{k}$ of projective lines. In [7], Hara and Watanabe gave a purely algebraic proof of the strong

\footnotetext{
${ }^{\dagger}$ Current address: Department of Mathematics, Meijo University, Nagoya 468-8502, Japan. Supported by Grant-in-Aid for Scientific Research.
} 
Lefschetz property the algebra $k\left[x_{1}, \ldots, x_{n}\right] /\left(x_{1}^{2}, \ldots, x_{n}^{2}\right)$ based on recursive formulas for determinants of some matrices. We will give another proof.

The Sperner property of the vector space lattice $V(q, n)$ consisting of the linear subspaces of the vector space $\mathbb{F}_{q}^{n}$ can be deduced from the result on the rank of its incidence matrices due to Kantor [9]. We will give another proof of the Sperner property of $V(q, n)$ based on the strong Lefschetz property of a Gorenstein algebra.

Our construction can be done for general matroids. For a matroid $M$ and its bases $\mathcal{B}$, we introduce a polynomial $\Phi_{M}:=\sum_{B \in \mathcal{B}} x_{B}$. The Gorenstein algebra $A_{M}$ will be defined to be the quotient algebra of the ring of the differential polynomials by the annihilator of $\Phi_{M}$. We show that the Gorenstein algebra $A_{M}$ associated to a modular geometric lattice has the strong Lefschetz property.

The organization of this article is the following: In 2.1. we will recall the definition of the Sperner property of the ranked posets. In 2.2, we will summarize some basic facts of matroids. We also will summarize basic facts of Gorenstein algebras in 3.1. Then we will introduce ideals associated to the matroids in 3.2. and state some results for genera matroids in 3.3 . In 4.1, 4.2 and 4.3, we will consider the cases related to the Boolean lattice, the vector space lattice, and the incidence lattice of a finite projective planes, and show some results for these special case.

This article is a research announcement of the recent results by the authors. Please see [11], the full version of this article, for more details.

\section{Posets associated to matroids}

\subsection{Ranked poset and Sperner property}

Here we recall the definition of the Sperner property.

We consider a finite poset $P$ with rank decomposition $\coprod_{i=0}^{m} P_{i}$, namely, for each element $v \in P_{i}$, the length of any saturated chain from a minimal element to $v$ is $i$.

Definition 2.1 A finite ranked poset $P=\coprod_{i>0} P_{i}$ is said to have the Sperner property if the maximal cardinality of antichains of $P$ is equal to $\max _{i}\left(\# P_{i}\right)$.

For a given ranked poset $P=\coprod_{i} P_{i}$, let $V_{i}$ be the vector space over some field spanned by the elements of $P_{i}$. The Sperner property for $P$ can be shown by constructing a sequence $\left(f_{0}, f_{1}, f_{2}, \ldots\right)$ of linear maps $f_{i}: V_{i} \rightarrow V_{i+1}$ satisfying a certain condition. Let $A^{(i)}=\left(a_{u v}^{(i)}\right)_{u \in P_{i}, v \in P_{i+1}}$ be the matrix representing $f_{i}$, i.e., the matrix such that

$$
f_{i}(u)=\sum_{v \in P_{i+1}} a_{u v}^{(i)} v, \quad u \in P_{i}
$$

If every matrix $A^{(i)}$ satisfies the condition $a_{u v}^{(i)} \neq 0 \Longrightarrow u<v$, and is of full rank, then $P$ has the Sperner property. (See e.g. [8] for details.)

Remark 2.2 Let $[n]:=\{1,2, \ldots, n\}$ be an $n$-element set. The set $P=2^{[n]}$ of the subsets of $[n]$ has a natural lattice structure induced by the operations $\cup$ and $\cap$. The obtained lattice is called the Boolean lattice. In [14], Sperner showed that

$$
\{\text { antichains of the maximum size }\}= \begin{cases}\left\{P_{n / 2}\right\} & \text { if } n \text { is even } \\ \left\{P_{(n-1) / 2}, P_{(n+1) / 2}\right\} & \text { if } n \text { is odd. }\end{cases}
$$


Hence the Boolean lattice has the Sperner property.

Remark 2.3 The Sperner property of the vector space lattice $V(q, n)$ can be deduced from the nondegeneracy of its incidence matrices due to Kantor [9].

Remark 2.4 In [1], it was proved the Sperner property of the finite lattice such that the number of elements covered by each element $v$ depend only on the rank of $v$, and the number of elements covering each element $v$ depend only on the rank of $v$. Many lattices satisfy the assumption, e.g., the Boolean lattice $2^{[n]}$, the vector space lattice $V(q, n)$, and the incidence lattice of a finite projective planes.

\subsection{Posets associated to matroids}

Definition 2.5 A pair $(E, \mathcal{F})$ of a finite set $E$ and $\mathcal{F} \subset 2^{E}$ is called a matroid if it satisfies the following axioms:

- $\emptyset \in \mathcal{F}$.

- If $X \in \mathcal{F}$ and $Y \subset X$, then $Y \in \mathcal{F}$.

- If $X, Y \in \mathcal{F}$ and $\# X>\# Y$, then there exists an element $x \in X \backslash Y$ such that $Y \cup\{x\} \in \mathcal{F}$.

Here, $\mathcal{F}$ is called the system of independent sets.

Definition 2.6 Let $M=(E, \mathcal{F})$ be a matroid.

- A maximal element $B \in \mathcal{F}$ is called a basis of $M$. The set of bases of $M$ is denoted by $\mathcal{B}=$ $\mathcal{B}(M) \subset \mathcal{F}$.

- For a subset $S \subset E$, define $r(S):=\max \{\# F \mid F \in \mathcal{F}, F \subset S\}$. The map $r: 2^{E} \rightarrow \mathbb{Z}$ is called the rank function of $M$.

- For a subset $S \subset E$, define the closure $\sigma(S)$ of $S$ by $\sigma(S):=\{y \in E \mid r(S \cup\{y\})=r(S)\}$. We define an equivalence relation $\sim$ on $2^{E}$ by $S \sim T \Longleftrightarrow \sigma(S)=\sigma(T)$.

Example 2.7 Let $E=[n]$ and $\mathcal{F}=2^{E}$. Then $(E, \mathcal{F})$ is a matroid. We denote the matroid by $M([n])$. In this case, every subset $S \subset E$ is a independent set. Hence $r(S)=\# S$ and $\sigma(S)=S$.

Example 2.8 The projective space $\mathbb{P}:=\mathbb{P}^{n-1}\left(\mathbb{F}_{q}\right)$ over a finite field $\mathbb{F}_{q}$ has the structure of a matroid by the usual linear independence. More precisely, if we define the system of independence set $\mathcal{F}$ by

$$
\mathcal{F}:=\left\{F \in 2^{\mathbb{P}} \mid F \text { is linearly independent over } \mathbb{F}_{q}\right\},
$$

then $(\mathbb{P}, \mathcal{F})$ is a matroid. We denote it by $M(q, n)$. In this case, the closure $\sigma(S)$ of a subset $S \in \mathbb{P}$ coincides with the linear subspace $\langle S\rangle$ of $\mathbb{P}$ spanned by $S$.

Lemma 2.9 Let $S, T \in \mathcal{F}$. Then we have

$$
S \sim T \Longleftrightarrow\{U \in \mathcal{F} \mid U \cap S=\emptyset, U \cup S \in \mathcal{F}\}=\{U \in \mathcal{F} \mid U \cap T=\emptyset, U \cup T \in \mathcal{F}\} .
$$


Let $M=(E, \mathcal{F})$ be a matroid, and $\mathcal{F}_{i} \subset \mathcal{F}$ for $i=0,1, \ldots, r(E)$, the set of independent sets of cardinality $i$, i.e.,

$$
\mathcal{F}_{i}:=\{F \in \mathcal{F} \mid \# F=i\} .
$$

Let $\Omega:=2^{E} / \sim$, and $\overline{\mathcal{F}}_{l}:=\mathcal{F}_{l} / \sim$. We can identify $\Omega$ with the set of flats $S \subset E$, i.e., subsets $S$ of $E$ such that $S=\sigma(S)$. Under this identification, we define the subset $\Omega(l), l=1, \ldots, r(E)$, of $\Omega$ by

$$
\Omega(l):=\left\{S \in 2^{E} \mid S=\sigma(S), r(S)=l\right\} .
$$

The set $\Omega=\coprod_{l} \Omega(l)$ ordered by the inclusion $\subset$ is a ranked poset.

Example 2.10 For the matroid $M([n])=\left([n], 2^{[n]}\right)$ defined in Example 2.7, $\Omega$ is the Boolean lattice $2^{[n]}$.

Example 2.11 For the matroid $M(q, n)$ defined in Example 2.8, $\Omega$ is the vector space lattice $V(q, n)$.

\section{Ideals associated to matroids}

\subsection{Basic facts of Gorenstein algebras}

Definition 3.1 Let $A=\bigoplus_{d=0}^{D} A_{d}$, where $A_{D} \neq 0$, be a graded Artinian algebra. We call an element $L \in A_{1}$ a strong Lefschetz element of $A$ if the multiplication map

$$
\times L^{D-2 i}: A_{i} \rightarrow A_{D-i}
$$

is bijective for $i=0, \ldots,[D / 2]$. We say that $A$ has the strong Lefschetz property (in the narrow sense) if there exists a strong Lefschetz element of $A$.

In the rest of this paper, we consider the Gorenstein algebras that is finite-dimensional over a field $k$ of characteristic zero.

Definition 3.2 [[13, Chapter 5, 6.5]] Let $A=\bigoplus_{d=0}^{D} A_{d}$ be a graded $k$-algebra that is finite-dimensional over $k$. We call $A$ the Poincaré duality algebra if $\operatorname{dim}_{k} A_{D}=1$ and the bilinear pairing

$$
A_{d} \times A_{D-d} \rightarrow A_{D} \cong k
$$

is non-degenerate for $d=0, \ldots,[D / 2]$.

The following is a well-known fact (see e.g. [4], [8], [10]).

Proposition 3.3 A graded Artinian k-algebra $A$ is a Poincaré duality algebra if and only if $A$ is Gorenstein.

Let $P=k\left[x_{1}, \ldots, x_{n}\right]$ and $Q=k\left[X_{1}, \ldots, X_{n}\right]$ be polynomial rings over $k$. We may regard $P$ as a $Q$-module via the identification $X_{i}=\partial / \partial x_{i}, i=1, \ldots, n$. For a polynomial $F(x) \in P$, denote by Ann $F$ the ideal of $Q$ generated by the differential polynomials annihilating $F$, i.e.,

$$
\text { Ann } F:=\{\varphi(X) \in Q \mid \varphi(X) F(x)=0\} .
$$

The following is immediate from the theory of the inverse systems (see [2], [3], [5]). 
Proposition 3.4 Let I be an ideal of $Q=k\left[X_{1}, \ldots, X_{n}\right]$ and $A=Q / I$ the quotient algebra. Denote by $\mathfrak{m}$ the maximal ideal $\left(X_{1}, \ldots, X_{n}\right)$ of $Q$. Then $\sqrt{I}=\mathfrak{m}$ and the $k$-algebra $A$ is Gorenstein if and only if there exists a polynomial $F \in R=k\left[x_{1}, \ldots, x_{n}\right]$ such that $I=\operatorname{Ann}_{Q} F$.

Example 3.5 The coinvariant algebra $R_{W}$ of the finite Coxeter group $W$ is an example of the Gorenstein algebra with the strong Lefschetz property. The coinvariant algebra $R_{W}$ is defined to be a quotient of the ring of polynomial functions on the reflection representation $V$ of $W$ by the ideal generated by the fundamental $W$-invariants. When $W$ is crystallographic (i.e., Weyl group), the Lefschetz property of $R_{W}$ is a consequence of the Hard Lefschetz Theorem for the corresponding flag variety $G / B$. Stanley [15] has shown the Sperner property of the strong Bruhat ordering on $W$ from the Lefschetz property of $R_{W}$ (except for type $H_{4}$ ). The Lefschetz property of $R_{W}$ of type $H_{4}$ has been confirmed in [12]. Since $R_{W}$ is Gorenstein, it has a presentation as in Proposition 3.4. In fact, $R_{W}$ is isomorphic to the algebra Sym $V^{*} / \operatorname{Ann} F$, where $F$ is the product of the positive roots.

Definition 3.6 Let $G$ be a polynomial in $k\left[x_{1}, \ldots, x_{n}\right]$. When a family $\mathbf{B}_{d}=\left\{\alpha_{i}^{(d)}\right\}_{i}$ of homogeneous polynomials of degree $d>0$ is given, we call the polynomial

$$
\operatorname{det}\left(\left(\alpha_{i}^{(d)}(X) \alpha_{j}^{(d)}(X) G(x)\right)_{i, j=1}^{\# \mathbf{B}_{d}}\right) \in k\left[x_{1}, \ldots, x_{n}\right]
$$

the $d$-th Hessian of $G$ with respect to $\mathbf{B}_{d}$, and denote it by $\operatorname{Hess}_{\mathbf{B}_{d}}^{(d)} G$. We denote the $d$-th Hessian simply by $\operatorname{Hess}^{(d)} G$ if the choice of $\mathbf{B}_{d}$ is clear.

When $d=1$ and $\alpha_{j}^{(1)}(X)=X_{j}, j=1, \ldots, n$, the first Hessian Hess ${ }^{(1)} G$ is the usual Hessian:

$$
\operatorname{Hess}^{(1)} G=\operatorname{Hess} G:=\operatorname{det}\left(\frac{\partial^{2} G}{\partial x_{i} \partial x_{j}}\right)_{i j} .
$$

Let a graded Gorenstein $k$-algebra $A=\bigoplus_{d} A_{d}$ be finite-dimensional over $k$. Take a polynomial $F \in Q$ such that $A=Q / \operatorname{Ann}_{Q} F$. The following gives a criterion for an element $L \in A_{1}$ to be a Lefschetz element.

Proposition 3.7 ([17, Theorem 4]) Fix an arbitrary k-linear basis $\mathbf{B}_{d}$ of $A_{d}$ for $d=1, \ldots,[D / 2]$. An element $L=a_{1} X_{1}+\cdots+a_{n} X_{n} \in A_{1}$ is a strong Lefschetz element of $A=Q / \operatorname{Ann}_{Q} F$ if and only if $F\left(a_{1}, \ldots, a_{n}\right) \neq 0$ and $\left(\operatorname{Hess}_{\mathbf{B}_{d}}^{(d)} F\right)\left(a_{1}, \ldots, a_{n}\right) \neq 0$ for $d=1, \ldots,[D / 2]$.

Corollary 3.8 If one of the Hessians $\operatorname{Hess}_{\mathbf{B}_{d}}^{(d)} F, d=1, \ldots,[D / 2]$, is identically zero, then $Q / \operatorname{Ann}_{Q} F$ does not have the strong Lefschetz property.

\subsection{Ideals associated to matroids}

We introduce ideals of $Q$ associated to matroids. Fix a matroid $M=(E, \mathcal{F})$ and a polynomial ring $Q=k\left[x_{e} \mid e \in E\right]$ in $\# E$ variables. We define the subsets $\mathcal{G}_{M}^{(0)}, \mathcal{G}_{M}^{(1)}$ and $\mathcal{G}_{M}^{(2)}$ of $k\left[x_{e} \mid e \in E\right]$ by

$$
\mathcal{G}_{M}^{(0)}=\left\{x_{e}^{2} \mid e \in E\right\}, \quad \mathcal{G}_{M}^{(1)}=\left\{x_{S} \mid S \notin \mathcal{F}\right\}, \quad \mathcal{G}_{M}^{(2)}=\left\{x_{A}-x_{A^{\prime}} \mid A, A^{\prime} \in \mathcal{F}, A \sim A^{\prime}\right\} .
$$


Let $\Lambda_{M}=\mathcal{G}_{M}^{(0)} \cup \mathcal{G}_{M}^{(1)} \cup \mathcal{G}_{M}^{(2)}$. We define $J_{M}$ to be the ideal of $k\left[x_{e} \mid e \in E\right]$ generated by $\Lambda_{M}$. For an equivalence class $\tau \in \Omega$, consider a polynomial $f_{\tau}$ given by

$$
f_{\tau}:=\sum_{F \in \mathcal{F} \cap \tau} x_{F}
$$

where $x_{F}:=\prod_{e \in F} x_{e}$. We also define a polynomial $\Phi_{M} \in k\left[x_{e} \mid e \in E\right]$ by

$$
\Phi_{M}:=\sum_{B \in \mathcal{B}} x_{B}
$$

For a equivalent class $\tau \in \Omega$ of $B \in \mathcal{B}$, it follows by definition that $\Phi_{M}=f_{\tau}$.

\subsection{Some results}

We consider the ideals $J_{M}, \operatorname{Ann}_{Q} \Phi_{M}$ and $\bigcap_{\tau \in \Omega} \operatorname{Ann} f_{\tau}$. We omit their proof. Please see [11] for the proof.

Theorem 3.9 For a matroid $M$, we have the following:

- The ideal Ann $\Phi_{M}$ contains $J_{M}$.

- The ideal $\bigcap_{\tau \in \Omega}$ Ann $f_{\tau}$ equals $J_{M}$.

- The subset $\Lambda_{M}$ of $Q$ is a universal Gröbner basis of $J_{M}$.

Corollary 3.10 The Hilbert polynomial of $Q / J_{M}$ is given by

$$
\operatorname{Hilb}\left(Q / J_{M}, t\right)=\sum_{i=0}^{r(E)}\left(\# \overline{\mathcal{F}}_{i}\right) t^{i} .
$$

Example 3.11 Let $M$ be a matroid defined by the following vectors.

\begin{tabular}{c|c|c|c|c}
$v_{1}$ & $v_{2}$ & $v_{3}$ & $v_{4}$ & $v_{5}$ \\
1 & 0 & 0 & 1 & 0 \\
0 & 1 & 0 & 1 & 1 \\
0 & 0 & 1 & 0 & 1
\end{tabular}

Then the set $\mathcal{B}$ of bases is $\{\{1,2,3\},\{1,2,5\},\{1,3,4\},\{1,3,5\},\{1,4,5\},\{2,3,4\},\{2,4,5\},\{3,4,5\}\}$. The ideal Ann $\Phi_{M}$ contains an additional generator other than $\Lambda_{M}$. In fact, we have

$$
\operatorname{Ann} \Phi_{M}=J_{M}+\left(x_{13}+x_{45}-x_{15}-x_{34}\right) .
$$

The Hilbert series of $Q / \operatorname{Ann} \Phi_{M}$ is $(1,5,5,1)$ and that of $Q / J_{M}$ is $(1,5,6,1)$. In particular, $Q / J_{M}$ is not Gorenstein. By direct computation, we obtain Hess $\Phi_{M}=8\left(x_{1}+x_{4}\right)\left(x_{3}+x_{5}\right) \Phi_{M}$. This implies that $Q / \operatorname{Ann} \Phi_{M}$ has the Lefschetz property. 


\section{Modular geometric lattices}

In this section, we consider three examples of lattices, the Boolean lattice, the vector space lattice and the incidence lattice of a projective plane. As in Example 3.11, the equality Ann $\Phi_{M}=J_{M}$ does not hold for a general matroid. The equation, however, holds in the three examples we discuss here. In 4.1, we consider the Boolean lattice. It is known that the algebra associated to the Boolean lattice has the strong Lefschetz property. We give another proof of the strong Lefschetz property for the algebra associated to the Boolean lattice. In a similar manner to our proof, we can prove the strong Lefschetz property for the algebras associated to the vector space lattice and the incidence lattice of a projective plane.

\subsection{The Boolean lattice}

For a matroid $M$ we define polynomials $\Phi_{M}^{(i)}:=\sum_{G \in \mathcal{F}_{i}} x_{G}$ and the matroid $M^{(i)}:=\left(E, \bigcup_{k=0}^{i} \mathcal{F}_{k}\right)$ for $i=1, \ldots, n$. Note that $\Phi_{M}^{(n)}=\Phi_{M}$ and that $\Phi_{M}^{(i)}=\Phi_{M^{(i)}}$. The polynomials $\Phi_{M}^{(i)}$ and the matroids $M^{(i)}$ play an important role. For $B \in \mathcal{F}_{l}$ and $0 \leq i \leq l$, define

$$
\mathcal{F}_{l}(B, i):=\left\{A \in \mathcal{F}_{l} \mid r(\sigma(A) \cap \sigma(B))=i\right\} .
$$

Then we have $\mathcal{F}_{l}(B, l)=\left\{A \in \mathcal{F}_{l} \mid A \sim B\right\}$ and $\mathcal{F}_{l}=\coprod_{i=0}^{l} \mathcal{F}_{l}(B, i)$. For $A, B \in \mathcal{F}_{l}$, define

$$
\mathcal{F}_{l}^{A}(B, i):=\left\{A^{\prime} \in \mathcal{F}_{l}(B, i) \mid A \cup A^{\prime} \in \mathcal{F}_{2 l}\right\} .
$$

For $B \in \mathcal{F}_{l}$, consider a polynomial $\Phi(B, i):=\sum_{A \in \mathcal{F}_{l}(B, i)} x_{A}$ and a differential polynomial $P(B, i):=$ $\sum_{A \in \mathcal{F}_{l}(B, i)} \partial^{A}$. Then it follows from straightforward calculation that

$$
P(B, i) \Phi_{M}^{(2 l)}=\sum_{j=0}^{l} \sum_{A^{\prime} \in \mathcal{F}_{l}(B, j)} \# \mathcal{F}_{l}^{A^{\prime}}(B, i) x_{A^{\prime}} .
$$

Now we consider the matroid $M([n])$ and $B \in \mathcal{F}_{l}$. In this case

$$
\begin{gathered}
\mathcal{F}_{l}=\left\{A \in 2^{[n]} \mid \# A \cap B=i\right\} \\
\mathcal{F}_{l}^{A}(B, i)=\left\{\begin{array}{l|c}
A^{\prime} \in 2^{[n]} & \begin{array}{c}
\# A^{\prime} \cap B=i, \\
\# A \cap A^{\prime}=0 \\
\# A^{\prime}=l
\end{array}
\end{array}\right\} .
\end{gathered}
$$

For $A \in \mathcal{F}_{l}(B, j)$, i.e., $A \in 2^{[n]}$ such that $\# A=l$ and $\# A \cap B=j$, we can obtain

$$
\# \mathcal{F}_{l}^{A}(B, i)=\left(\begin{array}{c}
\#[n] \backslash(A \cup B) \\
l-i
\end{array}\right)\left(\begin{array}{c}
\# B \backslash A \\
i
\end{array}\right)=\left(\begin{array}{c}
n-2 l+j \\
l-i
\end{array}\right)\left(\begin{array}{c}
l-j \\
i
\end{array}\right) .
$$

Hence $\# \mathcal{F}_{l}^{A}(B, i)$ does not depend on the choice of $A \in \mathcal{F}_{l}(B, j)$. Let $a_{i j}^{(l)}=\left(\begin{array}{c}n-2 l+j \\ l-i\end{array}\right)\left(\begin{array}{c}l-j \\ i\end{array}\right)$, and consider the matrix $\left(a_{i, l-j}^{(l)}\right)_{i, j=0}^{l}$. Then

$$
\left(\begin{array}{c}
P(B, 0) \Phi_{M}^{(2 l)} \\
\vdots \\
P(B, l) \Phi_{M}^{(2 l)}
\end{array}\right)=\left(a_{i, l-j}^{(l)}\right)_{i, j=0}^{l}\left(\begin{array}{c}
\Phi(B, l) \\
\vdots \\
\Phi(B, 0)
\end{array}\right) .
$$


It is easy to show that the matrix is upper-triangular, and that $a_{i, l-i}^{(l)}=\left(\begin{array}{c}n-l-i \\ l-i\end{array}\right)$. Hence if $n \geq 2 l$, then the determinant $\operatorname{det}\left(a_{i, l-j}^{(l)}\right)_{i, j=0}^{l}=\prod_{i=0}^{l}\left(\begin{array}{c}n-l-i \\ l-i\end{array}\right)$ is positive. Since the matrix is invertible, $\Phi_{M}(B, l)$ is written as a linear combination of $P(B, 0) \Phi_{M}^{(2 l)}, P(B, 1) \Phi_{M}^{(2 l)}, \ldots, P(B, l) \Phi_{M}^{(2 l)}$. In other words, there exists a differential polynomial $P_{B}$ such that $\Phi_{M}(B, l)=P_{B} \Phi_{M}^{(2 l)}$. Since $\mathcal{F}_{l}(B, l)=\left\{A \in \mathcal{F}_{l} \mid A \sim B\right\}$, $\mathcal{F}_{l}(B, l)$ and $\mathcal{F}_{l}\left(B^{\prime}, l\right)$ are disjoint if $B \nsim B^{\prime}$. Hence $\left\{\Phi_{M}(B, l)\right\}$ spans $\# \overline{\mathcal{F}}_{l}$-dimensional $k$-vector space. Since Ann $\Phi_{M^{(2 l)}}$ contains $J_{M^{(2 l)}}$, we have the following lemma:

Lemma 4.1 Let $M=M([n])$ and $2 l \leq n$. Then $\left\{\partial^{F} \Phi_{M}^{(2 l)} \mid F \in \overline{\mathcal{F}}_{l}\right\}$ spans \# $\overline{\mathcal{F}}_{l}$-dimensional $k$-vector space.

Take representatives $F_{1}, \ldots, F_{m_{l}} \in \mathcal{F}_{l}$ of $\overline{\mathcal{F}}_{l}$. For $F, F^{\prime} \in \mathcal{F}_{l}$, define

$$
\delta_{F, F^{\prime}}:= \begin{cases}1, & \text { if } \sigma(F) \cap \sigma\left(F^{\prime}\right)=\emptyset, \\ 0, & \text { otherwise }\end{cases}
$$

It follows from direct calculation that

$$
\operatorname{det}\left(\partial^{F_{i}} \partial^{F_{j}} \Phi_{M}^{(2 l)}\right)_{i, j=1}^{m_{l}}=\operatorname{det}\left(\delta_{F_{i}, F_{j}}\right)_{i, j=1}^{m_{l}} .
$$

The algebra $B^{(2 l)}:=Q / \operatorname{Ann} \Phi_{M}^{(2 l)}$ is Gorenstein, and the natural pairings

$$
\langle,\rangle: B_{i}^{(2 l)} \times B_{2 l-i}^{(2 l)} \rightarrow B_{2 l}^{(2 l)} \cong k
$$

are non-degenerate for $i=0, \ldots, l$. In the case of $M([n])$, it follows from Lemma 4.1 that the dimension of the homogeneous space of of $B_{l}^{(2 l)}$ of degree $l$ is $\# \overline{\mathcal{F}}_{l}$. Hence we have the following:

Lemma 4.2 If $n \geq 2 l$, then we have $\operatorname{det}\left(\delta_{F_{i}, F_{j}}\right)_{i, j=1}^{m_{l}} \neq 0$.

Let $M=M([n])$. Then, for $F \in \mathcal{F}_{j}$, \#\{ $\left.F^{\prime} \in \mathcal{F}_{i} \mid F \cup F^{\prime} \in \mathcal{F}_{i+j}\right\}=\left(\begin{array}{c}n-j \\ i\end{array}\right)$, which does not depend on the choice of $F \in \mathcal{F}_{j}$. Hence, for $k \geq 2 l$, the determinant of the matrix

$$
\left.\left(\partial^{F_{i}} \partial^{F_{j}} \Phi_{M}^{(k)}\right)_{i, j=1}^{m_{l}}\right|_{x=1}
$$

equals $\gamma \operatorname{det}\left(\delta_{F_{i}, F_{j}}\right)_{i, j}^{m_{l}}$ for some $\gamma \neq 0$. Therefore the matrix $\left(\partial^{F_{i}} \partial^{F_{j}} \Phi_{M}^{(k)}\right)_{i, j=1}^{m_{l}}$ is not identically zero. Hence we have the following:

Theorem 4.3 Let $M=M([n])$ and $k \leq n$. Then we have the following:

- The polynomial $\Phi_{M^{(k)}}$ equals the elementary symmetric function $e_{k}\left(x_{1}, \ldots, x_{n}\right)$.

- The ideal Ann $\Phi_{M}$ equals $J_{M}$.

- The Gorenstein algebra $Q / \operatorname{Ann} \Phi_{M^{(k)}}=Q / \operatorname{Ann} e_{k}\left(x_{1}, \ldots, x_{n}\right)$ has the strong Lefschetz property. 
- The Hilbert series of the algebra $Q / \operatorname{Ann} \Phi_{M(k)}$ satisfies

$$
\operatorname{Hilb}\left(Q / \operatorname{Ann} \Phi_{M^{(k)}}, t\right)=\sum_{i \leq k / 2}\left(\begin{array}{c}
n \\
i
\end{array}\right) t^{i}+\sum_{i>k / 2}\left(\begin{array}{c}
n \\
k-i
\end{array}\right) t^{i} .
$$

- The poset $\Omega$ has the Sperner property. Or equivalently the Boolean lattice has the Sperner property.

\subsection{The vector space lattice}

Here we consider the matroid $M=M(q, n)$ defined in Example 2.8 In a similar manner to the case of $M([n])$, we can prove the strong Lefschetz property for the Gorenstein algebra associated to the matroid $M(q, n)$. Since the same argument works, we give only the sketch of the proof. See [11] for more details.

Similarly to the case of $M([n]), a_{i j}^{(l)}=\# \mathcal{F}_{l}^{A}(B, i)$ does not depend on the choice of $A \in \mathcal{F}_{l}(B, j)$, and the matrix $\left(a_{i, l-j}^{(l)}\right)_{i, j=0}^{l}$ is invertible. Hence we have the following lemma:

Lemma 4.4 Let $M=M(q, n)$ and $2 l \leq n$. Then $\left\{\partial^{F} \Phi_{M}^{(2 l)} \mid F \in \overline{\mathcal{F}}_{l}\right\}$ spans $\# \overline{\mathcal{F}}_{l}$-dimensional $k$ vector space.

Moreover \# $\left\{F^{\prime} \in \mathcal{F}_{i} \mid F \cup F^{\prime} \in \mathcal{F}_{i+j}\right\}$ is also independent of the choice of $F \in \mathcal{F}_{j}$. Hence the lemma implies the following:

Theorem 4.5 Let $M=M(q, n)$ and $k \leq n$. Then we have the following:

- The ideal Ann $\Phi_{M}$ equals $J_{M}$.

- The Gorenstein algebra $Q / \operatorname{Ann} \Phi_{M^{(k)}}$ has the strong Lefschetz property.

- The Hilbert series of the algebra $Q / \operatorname{Ann} \Phi_{M^{(k)}}$ satisfies

$$
\operatorname{Hilb}\left(Q / \operatorname{Ann} \Phi_{M^{(k)}}, t\right)=\sum_{i \leq k / 2}\left[\begin{array}{c}
n \\
i
\end{array}\right]_{q} t^{i}+\sum_{i>k / 2}\left[\begin{array}{c}
n \\
k-i
\end{array}\right]_{q} t^{i},
$$

where $\left[\begin{array}{l}n \\ i\end{array}\right]_{q}$ stands for a q-binomial coefficient.

- The poset $\Omega$ has the Sperner property. Or equivalently, the vector space lattice $V(q, n)$ consisting of the linear subspaces of $\mathbb{F}_{q}^{n}$ has the Sperner property.

Remark 4.6 We can obtain a universal Gröbner basis for the ideal Ann $\Phi_{M(q, n)}$ by Theorems 3.9 and 4.5. In [11], the Gröbner fan of the ideal is also discussed. The Gröbner fan $G\left(\operatorname{Ann} \Phi_{M(q, n)}\right)$ can be recovered from the tropical hypersurfaces of certain polynomials defined by the bases of the linear subspaces of $\mathbb{P}^{n-1}\left(\mathbb{F}_{q}\right)$.

\subsection{The incidence lattice of a finite projective plane}

Here we consider a matroid $M=(E, \mathcal{F})$ such that $r(E)=3, \# E=\# \overline{\mathcal{F}}_{1}=\# \overline{\mathcal{F}}_{2}$, and $\# \sigma(S)=N$ for all $S \in \mathcal{F}_{2}$. The assumption $\# E=\# \overline{\mathcal{F}}_{1}$ implies $\mathcal{F}_{2}=\{S \subset E \mid \# S=2\}$. Hence, for $v, w \in E$, 
$\partial_{v} \partial_{w} \Phi_{M}=0$ if and only if $v=w$. Moreover $\left.\partial_{v} \partial_{w} \Phi_{M}\right|_{x=1}=N$ for $v \neq w \in E$ since $\# \sigma(S)=N$ for all $S \in \mathcal{F}_{2}$. It follows that

$$
\left.\left(\partial_{v} \partial_{w} \Phi_{M}\right)_{v, w \in E}\right|_{x=1}=N^{\# E}\left(\partial_{v} \partial_{w} e_{2}(x)\right)_{v, w \in E}=N^{\# E}\left(\partial_{v} \partial_{w} \Phi_{M^{(2)}}\right)_{v, w \in E} .
$$

Theorem 4.3 implies that the determinant of the matrix is not identically zero. Hence we have the following:

Theorem 4.7 Let $M=(E, \mathcal{F})$ be a matroid such that $r(E)=3$, $\# E=\# \overline{\mathcal{F}}_{1}=\# \overline{\mathcal{F}}_{2}$, and $\# \sigma(S)=N$ for all $S \in \mathcal{F}_{2}$. Then we have the following:

- The ideal Ann $\Phi_{M}$ equals $J_{M}$.

- The Gorenstein algebra $Q / J_{M}$ has the strong Lefschetz property.

- The Hilbert series of the algebra $Q / \operatorname{Ann} \Phi_{M}$ is $1+(\# E) t+(\# E) t^{2}+t^{3}$.

- The poset $\Omega$ has the Sperner property.

Remark 4.8 Since the matroid $M(\Pi)$ associated to a finite projective plane $\Pi$ satisfies the conditions of Theorem 4.7 the incidence lattice of $\Pi$ has the Sperner property. The fundamental theorem of projective geometry shows that a modular geometric lattice decomposes into a direct product of Boolean lattices, vector space lattices, incidence lattices of finite projective planes, and lattices of rank 2 (see e.g. [16]). In [6], Greene has shown that a finite geometric lattice is modular if and only if the number of atoms equals the number of coatoms. It follows from this characterization a finite geometric lattice that the Gorenstein algebra $Q / J_{M}$ has the strong Lefschetz property if and only if the poset $\Omega$ associated to the matroid is a modular geometric lattice. See [11] for the detail.

\section{References}

[1] K. A. Baker, A generalization of Sperner's lemma, J. Combinatorial Theory 6 (1969), 224-225.

[2] W. Bruns and J. Herzog, Cohen-Macauley rings, Cambridge Studies in Advanced Mathematics, 39, Cambridge Univ. Press, Cambridge, 1993.

[3] A. V. Geramita, Inverse systems of fat points: Waring's problem, secant varieties of Veronese varieties and parameter spaces for Gorenstein ideals, in The Curves Seminar at Queen's, Vol. X, Queen's Papers in Pure and Appl. Math., 102, Queen's Univ., Kingston, ON, 1996, 2-114.

[4] A. V. Geramita, T. Harima, J. C. Migliore and Y. S. Shin, The Hilbert function of a level algebra, Mem. Amer. Math. Soc. 186 (2007), no. 872, 139 pp.

[5] S. Goto and K. Watanabe, On graded rings I, J. Math. Soc. Japan 30 (1978), 179-213.

[6] C. Greene, A rank inequality for finite geometric lattices, J. Combinatorial Theory, 9 (1970), 357364.

[7] M. Hara and J. Watanabe, The determinants of certain matrices arising from the Boolean lattice, Discrete Math. 308 (2008), no. 23, 5815-5822. 
[8] T. Harima, T. Maeno, H. Morita, Y. Numata, A. Wachi and J. Watanabe, The Lefschetz properties, (book manuscript), 2011, available at http://www.stat.t.u-tokyo.ac.jp/ numata/ tex/2010/hmmnww/

[9] W. M. Kantor, On incidence matrices of finite projective and affine spaces, Math. Z. 124 (1972), 315-318.

[10] T. Maeno and J. Watanabe, Lefschetz elements of Artinian Gorenstein algebras and Hessians of homogeneous polynomials, Illinois J. Math. 53 (2009), no. 2, 591-603.

[11] T. Maeno and Y. Numata, Sperner Property and Finite-dimensional Gorenstein Algebras Associated to Matroids, preprint. arXiv:1107.5094v2

[12] Y. Numata and A. Wachi, The strong Lefschetz property of the coinvariant ring of the Coxeter group of type $H_{4}$, J. Algebra 318 (2007), no. 2, 1032-1038.

[13] L. Smith, Polynomial Invariants of Finite Groups, Research Notes in Mathematics Vol. 6, A K Peters Ltd., 1995.

[14] E. Sperner, Ein Satz über Untermengen einer endlichen Menge, Math. Z. 27 (1928), no. 1, 544-548.

[15] R. P. Stanley, Weyl groups, the hard Lefschetz theorem and the Sperner property, Siam. J. Alg. and Disc. Meth. 1 (1980), 168-184.

[16] R. P. Stanley, An introduction to hyperplane arrangements, Geometric Combinatorics, 389-496, IAS/Park City Math. Ser., 13, AMS, Providence, RI, 2007.

[17] J. Watanabe, A remark on the Hessian of homogeneous polynomials, in The Curves Seminar at Queen's Volume XIII, Queen's Papers in Pure and Appl. Math., Vol. 119, 2000, 171-178. 
